\title{
An Evolutionary Study of Multi-Objective Workflow Scheduling in Cloud Computing
}

\author{
Shashank Shukla* \\ Anil Kumar Gupta** \\ Research Scholar \\ Department of IT*, CSE** \\ GCET Greater Noida UP
}

(INDIA)

\author{
Sandeep Saxena \\ Research Scholar \\ Department of CSE \\ NIT Durgapur INDIA
}

\author{
Santosh Kumar \\ Upadhyay \\ Asstt. Prof. \\ Department of CSE \\ GCET Greater Noida UP \\ (INDIA)
}

\begin{abstract}
Cloud computing become more popular in every field of life nowadays. This happened only due to its amazing services that provide to clients in the form of everything-as-aservice(XaaS). Where at one side cloud computing is gaining popularity and another side its faces some issues i.e. security issue, total cost issue, energy consumption issue, performance issue, QoS issue, etc. In above all challenges the quality of services is the most noticeable challenge and affects the cloud computing services. Quality of services can be improved by considering the several factors, scheduling of workload for suitable cloud computing resources one of them. If the cloud computing resources are scheduled accurately, it affects the response time of services, total cost of cloud resources, reduce the energy consumption, reduce the $\mathrm{CO} 2$ emission and enhance the performance of whole cloud system. In this paper, we characterize a comparative review of multiobjective workflow scheduling algorithms that are listed below.
\end{abstract}

\section{Keywords}

Cloud computing, multi-cloud computing, Grid-Computing, Multi-Objective workflow scheduling, workflow scheduling and QoS.

\section{INTRODUCTION}

Cloud Computing is a trendy model in Information Technology today. It provides lots of services instantly. As it becoming popular, it is also becoming more challenging and developing area of computing. It also provides a multi-objective resources that are newly developed and broad research area for researchers. Multiobjective is a complex optimization problem that requires considering different criteria so as to meet a large number of QoS (Quality of Service) requirements [3]. The Problem is, even more complex and challenging in workflow scheduling for conflicting requirements and goals. In this paper, our main goal is providing a comparative review of multi-objective workflow scheduling. Nowadays, the number of commercial cloud providers is rapidly increasing. In a commercial multi-cloud environment, individual providers are focused toward increasing their revenue and do not care about the utility of users and other providers. In such situation, we cannot faith the information offered by the providers. As the influence of the Internet in solving the large-scale problem is growing, the scenario of having multiple self-interested agents is becoming more and more popular [12]. The rest of paper organize as follows: Section-2 presents the multi-cloud introduction as well as its objective. Section-3 covers the related work presented by research scholar in this field. Section-4 represents the analytical summary of the survey and finally we conclude this paper with future work.

\section{MULTI-CLOUD}

In this modish era where mobile device becomes more needful gadget, in that Cloud Computing plays a main role by providing lots of services like everything-as-a-service (XaaS), including SaaS, PaaS, and IaaS. Cloud Computing enables a universal network access to stake platform of resources. Nowadays technology moving towards the multi-cloud world. Multi-cloud provides more than one cloud services in single assorted architecture. It used for reducing the risk of data loss due to localized component breakdown. It can also expand the total performance by using dissimilar infrastructures to serve the need of the miscellaneous customer and also by avoidance of 'vendor lock-in'.

Multi-cloud is mix up of multiple cloud computing services into a single architecture. This area provides more cloud services and decreases the risk of data loss. It can offer software, hardware and also necessary infrastructure to improve fault tolerance.

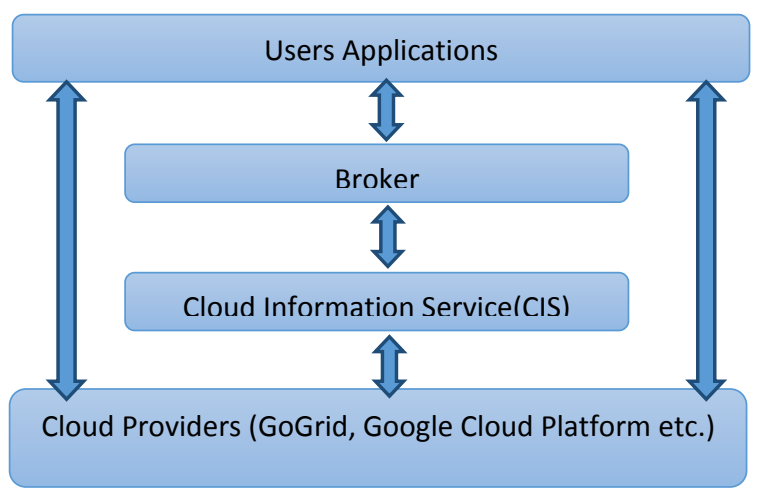

Figure-1. Multi-Cloud Architecture [12]

\subsection{MULTI-OBJECTIVE WORKFLOW SCHEDULING}

These days' enterprises and researchers antagonize with multiple competing objectives or goals like makespan in highperformance computing and economic cost in Cloud that have optimized simultaneously. Workflow scheduling, bigger task is divided into different-different small co-tasks these co-task or sub-tasks are allocated to resources in such a way that achieve some pre-define objective.[12] Usually, in multiobjective optimization problem can be defined as the problem of instantaneously increasing or decreasing of multiple conflicting goals.[1] Multi-objective scheduling is getting growing exploration attention. Multi-objective aggregate all the objectives in a single function defined a deductive without any knowledge about the problem being solved [2]. 


\section{LITERATURE SURVEY ON WORKFLOW SCHEDULING ALGORITHMS}

In the current scenario, many scientists/ researcher are working for improving the proper utilization of cloud resources. This problem is similar to some other environments like the grid or distributed. In the cloud computing environment, we can improve QoS with the help of workflow scheduling algorithms. We studied various algorithm, and some of them discussed here.

\section{A. Multi-Objective Approach for Energy-Aware} Workflow Scheduling in Cloud Computing Environments [3]: The main purpose of this paper is reducing the energy consumption of cloud computing resources by considering the multi-objectives of the quality of services(QoS). In the proposed work authors use a hybrid PSO algorithm to improve the scheduling performance (makespan, cost) and Dynamic Voltage and Frequency Scaling(DVFS) technique to minimize the energy consumption. Experiment and simulation of this approach done on two type of workflow namely neuroscience workflow and protein annotation workflow. DVFS-MODPSO give the better result in compare to HEFT algorithm [4] in the term of scheduling performance and also able to reduce the energy consumption.

B. A Multi-Objective Task Scheduling Algorithm for Heterogeneous Multi-Cloud Environment[5]: In this paper, authors proposed the two-phase multi-objective task scheduling (MOTS) algorithm for the heterogeneous multi-cloud environment. They have considered multiple objectives such as makespan time and execution cost. When proposed algorithm simulated with synthetic and benchmark data set it gives 48-time units, as makespan, 65 cost units, and $96.88 \%$ average cloud utilization. This experimental result is better when it compared with other two existing algorithms namely CMMS [6] and PBTS.

C. Multi-Objective Tasks Scheduling Algorithm for Cloud Computing Throughput Optimization [7]: In this algorithm author proposed multi-task scheduling algorithm that improves the data centre performance without violating SLA. The proposed algorithm used non-dominating sorting algorithm for solving the multiobjective (Task Size, QoS value). Maintaining a list of VM according to MIPS in descending order by cloud broker for selecting the VM one of them from a list. Updating of this list is done dynamically after a fixed time interval. When this algorithm compared with existing algorithm, then it gives optimize throughput.

D. A Multiple QoS Constrained Scheduling Strategy of Multiple Workflows for Cloud Computing [8]: In this paper, MengXu et.al. introduce a multiple QoS constrained scheduling algorithm for multiple workflow which schedule the multiple workflow that can be start at any time. They considered the four factors (QoS requirements, minimize makespan cost and success rate) that importantly affect the makespan time and cost of workflow. In this paper, author gives the better solution that previously proposed work such cost based scheduling algorithm introduced by Jia Yu [9] in which relationship between the workflow not considered. In other paper where $\mathrm{Ke} \mathrm{Liu} \mathrm{et} \mathrm{al,} \mathrm{[10]} \mathrm{proposed} \mathrm{for}$ intensive workflow (multiple instances of single workflow). When this algorithm simulated and compare with RANK_HYBD scheduling [11], then it gives improved success rate of the workflow.

E. Evolving Multi-Objective Strategies for Task Allocation of Scientific Workflows on Public Clouds[11]: EMST allocation of Scientific workflow proposed by Claudia and Trent in 2012, that allocate the scientific task on the public cloud with single and multiple objectives. As the single objective, it gives the similar result with algorithm particle swarm optimization for small workflow but when large workflow taken then it improves the outcome up to $80 \%$, similarly with multiple objectives also. Algorithm considers the workflow runtime, communication overhead, and overall execution cost as parameters. EMST improves the total workflow runtime, as well as the total execution cost of the workflow when It deployed on Amazon EC2 \& S3.

F. A Truthful Dynamic Workflow Scheduling Mechanism for Commercial Multi-Cloud Environments[12]: In this paper, The author proposed a new polynomial multi-objective scheduling for scientific workflow application in heterogeneous environments. This scheduling algorithm estimates optimal result using list scheduling heuristic with multi-objective optimization [13]. The top goal of this paper is maximizing the distance to constraint vector for the dominant solution. The new polynomial multi-objective scheduling algorithm is a part of ASKALON [14] and cloud infrastructures and Grids.

G. Multi-Objective Scheduling of many tasks in cloud platforms [15]: In this paper, the author proposed an optimized ordinal method for multi-objective many task scheduling that generates suboptimal or sufficiently good schedules for smooth multitask workflows on cloud platforms. Authors drove simulation based optimization and extend the ordinal optimization [16] [17] for cloud workflow scheduling. They achieved problem scalability on virtualized cloud platform that reduce half searching time than Monte Carlo and Blind Pick methods.

H. Multi-Objective Workflow Scheduling: An Analysis of the Energy Efficiency and Makespan Trade-off [18]: In this paper, author reformulates the problem as a bi-objective optimization through considering makespan and energy as goals. Author model workflow application as a direct acyclic graph/ interconnected through control flow and data flow dependencies and assumed hardware platform consist of set heterogeneous resources. They considered multi-core CPU as many resources reflected by ignoring overhead and using different cores in the same CPU for computing the makespan [19], and considering only two different levels of energy consumption: idle or fully loaded [18], [19]. The author achieved the result from $85 \%$ energy consumption and $3.3 \%$ of makespan concessions.

I. A Multi-Objective Approach for Workflow Scheduling in Heterogeneous Environments [20]: In this paper, the author proposed a new polynomial multiobjective scheduling algorithm for scientific work-flow. The algorithm approaches an optimal solution using a list scheduling heuristic joint with multi-objective optimization theory pointing two goals: maximizing the distance to the constraint vector for dominant solutions and minimizing it otherwise. 
J. Multi-objective QoS Optimization Based on Multiple Workflow Scheduling in Cloud Environment [21]: In this paper, the author evaluates performance for different -different test cases with a various number of workflows and an alternate set of QoS parameters by using MQMCE scheduling [22]. The author proposed system used MQMCE algorithm that satisfied the multiple QOS such as reducing cost. MQMCE algorithm used to reduce the cost, to reduce the time and increase the reliability and availability that results better than Round Robin Algorithm.

\section{ANALYSIS OF MULTI-WORKFLOW ALGORITHMS}

As we discussed in the above section, each multi-workflow algorithms consider one or multiple parameters by this, multiworkflow algorithms are enhanced by their own feature. Some algorithms are the focus on reducing the energy consumption whereas some proposed an optimized method. At the same time, multi-workflow scheduling algorithms try to achieve reduced cost and optimal solution. Each multiworkflow has the different structure that fit in their own types of cloud architecture and scheduling strategies. In given table we compare survey algorithm by dissimilar attributes.

\begin{tabular}{|c|c|c|c|c|c|c|}
\hline Name of Algorithm & Year & $\begin{array}{l}\text { Nature of } \\
\text { Algorithm }\end{array}$ & Objective & Environment & Criteria & $\underset{\mathbf{n}}{\text { Simulatio }}$ \\
\hline $\begin{array}{c}\text { A Multi-Objective Task Scheduling } \\
\text { Algorithm for Heterogeneous Multi-Cloud } \\
\text { Environment [5] }\end{array}$ & 2015 & Heuristic & $\begin{array}{l}\text { Make span, } \\
\text { Cost } \\
\text { and energy }\end{array}$ & Multi-cloud & Budget & MATLAB \\
\hline $\begin{array}{l}\text { Multi-Objective Tasks Scheduling } \\
\text { Algorithm for Cloud Computing } \\
\text { Throughput Optimization [7] }\end{array}$ & 2015 & Heuristic & $\begin{array}{c}\text { Execution time } \\
\text { Throughput }\end{array}$ & $\begin{array}{c}\text { Cloud } \\
\text { Computing }\end{array}$ & $\begin{array}{l}\text { Non- } \\
\text { dominated } \\
\text { sorting }\end{array}$ & CloudSim \\
\hline $\begin{array}{l}\text { Multi-objective scheduling of many tasks in } \\
\text { cloud platforms [15] }\end{array}$ & 2014 & Heuristic & $\begin{array}{l}\text { minimize the } \\
\text { makespan, } \\
\text { reducing the } \\
\text { resource cost, } \\
\text { preserving the } \\
\text { fault tolerance }\end{array}$ & $\begin{array}{c}\text { Cloud } \\
\text { computing, } \\
\text { Many-task } \\
\text { computing, } \\
\text { workflow }\end{array}$ & $\begin{array}{c}\text { Cluster } \\
\text { based }\end{array}$ & Real word \\
\hline $\begin{array}{c}\text { Truthful Dynamic Workflow Scheduling } \\
\text { Mechanism for Commercial Multi-Cloud } \\
\text { Environments [12] }\end{array}$ & 2013 & Heuristics & $\begin{array}{l}\text { Monetary cost, } \\
\text { Completion time }\end{array}$ & $\begin{array}{l}\text { Multi-cloud } \\
\text { Computing, } \\
\text { Game theory }\end{array}$ & $\begin{array}{c}\text { Structural } \\
\text { dependency, } \\
\text { Aggregation, } \\
\text { Complexity }\end{array}$ & Grid Sum \\
\hline $\begin{array}{c}\text { Multi-objective QoS Optimization Based } \\
\text { On Multiple Workflow Scheduling in Cloud } \\
\text { Environment [21] }\end{array}$ & 2013 & $\begin{array}{l}\text { Heuristic } \\
\text { and Genetic } \\
\text { algorithm }\end{array}$ & $\begin{array}{l}\text { time effect, cost } \\
\text { effect, } \\
\text { reliability }\end{array}$ & $\begin{array}{l}\text { Cloud } \\
\text { Computing, } \\
\text { Workflow, } \\
\text { QoS }\end{array}$ & $\begin{array}{c}\text { data } \\
\text { dependencies } \\
, \\
\text { budget } \\
\text { constrained }\end{array}$ & $\begin{array}{c}\text { Cloud } \\
\text { Sum }\end{array}$ \\
\hline $\begin{array}{c}\text { Multi-Objective Workflow Scheduling: An } \\
\text { Analysis of the Energy Efficiency and } \\
\text { Makespan Trade-off [18] }\end{array}$ & 2013 & Heuristic & $\begin{array}{c}\text { Optimizing } \\
\text { application, } \\
\text { Energy efficiency }\end{array}$ & $\begin{array}{c}\text { Cloud } \\
\text { computing, } \\
\text { Multi- } \\
\text { Workflow }\end{array}$ & $\begin{array}{l}\text { Makespan, } \\
\text { energy } \\
\text { consumption }\end{array}$ & $\begin{array}{c}\text { Real } \\
\text { World }\end{array}$ \\
\hline $\begin{array}{l}\text { Multi-Objective Approach for Energy- } \\
\text { Aware Workflow Scheduling in Cloud } \\
\text { Computing Environments [3] }\end{array}$ & 2013 & Heuristic & $\begin{array}{l}\text { Makespan, Cost, } \\
\text { Energy }\end{array}$ & $\begin{array}{c}\text { Cloud } \\
\text { Computing }\end{array}$ & $\begin{array}{l}\text { Interrelated } \\
\text { relationship } \\
\mathrm{b} / \mathrm{w} \text { factors }\end{array}$ & $\begin{array}{c}\text { Real } \\
\text { world \& } \\
\text { Synthetic } \\
\text { Data set }\end{array}$ \\
\hline $\begin{array}{c}\text { Evolving Multi-Objective Strategies for } \\
\text { Task Allocation of Scientific Workflows on } \\
\text { Public Clouds [11] }\end{array}$ & 2012 & Heuristic & $\begin{array}{l}\text { Improving the } \\
\text { Execution time, } \\
\text { cost in Single } \\
\text { Objective and } \\
\text { Multi-Objective }\end{array}$ & $\begin{array}{l}\text { Public Cloud } \\
\text { Computing }\end{array}$ & $\begin{array}{c}\text { Execution } \\
\text { cost, } \\
\text { Execution } \\
\text { runtime and } \\
\text { communicati } \\
\text { on overhead }\end{array}$ & $\begin{array}{l}\text { Amazon } \\
\text { EC2, S3 }\end{array}$ \\
\hline $\begin{array}{c}\text { A Multiple QoS Constrained Scheduling } \\
\text { Strategy of Multiple Workflows for Cloud } \\
\text { Computing [8] }\end{array}$ & 2009 & $\begin{array}{l}\text { Meta- } \\
\text { Heuristic }\end{array}$ & $\begin{array}{l}\text { Multi Workflow, } \\
\text { Makespan, Cost, } \\
\text { Success rate of } \\
\text { workflow }\end{array}$ & $\begin{array}{c}\text { Cloud } \\
\text { Computing }\end{array}$ & $\begin{array}{l}\text { minimum } \\
\text { surplus } \\
\text { time/cost \& } \\
\text { minimum } \\
\text { covariance }\end{array}$ & $\begin{array}{c}\text { Real } \\
\text { World }\end{array}$ \\
\hline
\end{tabular}




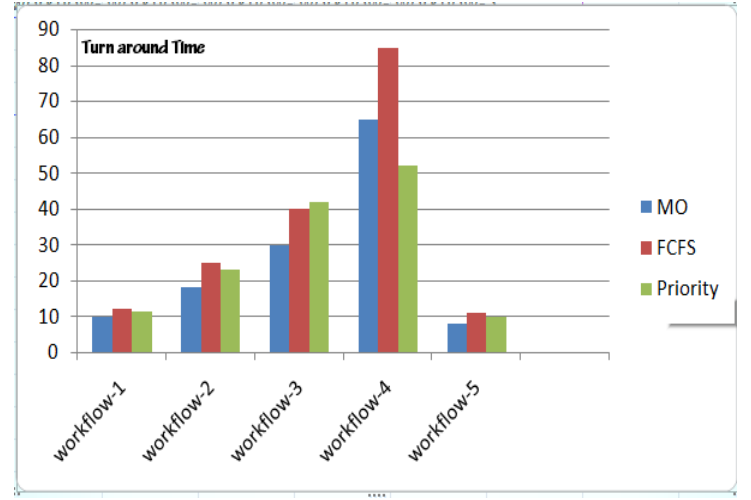

Graph-4.1

\section{DVFS-MODPSO[3]}

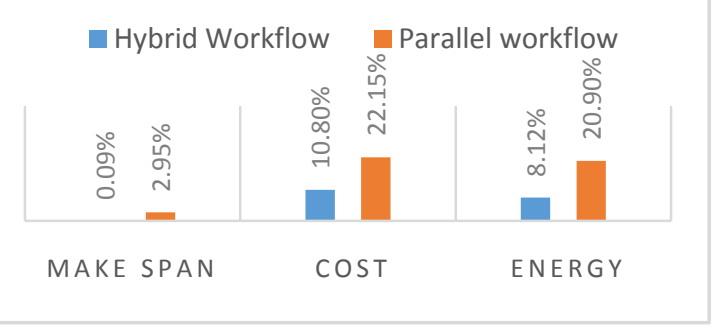

Graph-4.2

Graph 4.1 showing how the algorithm [7] gives better performance in the term of turnaround time whereas, in Graph-4.2 we can see clearly proposed algorithm [3] worked when it tested with the hybrid workflow.

\section{CONCLUSION AND FUTURE WORK}

On the base of beyond study, we had seen that algorithms established according to the user priority parameters. Parameters, which enhance security issue, total cost issue, energy consumption issue, performance issue, QoS issue in the area of Multi-objective workflow.

But still multi-cloud computing is not widely used due to lack of response time of services, total cost of cloud resources, reduce the energy consumption, reliability, availability and fault tolerance, etc., because of the third party and server locations. From time to time client data did not accessible due to huge quantity of multi-data request or network traffic with concern of that client faith has been fall rapidly. Sometimes resources allocated but client task did not do, and consistency had affected.

\section{REFERENCES}

[1] RituGarg, Awadhesh Kumar Singh "Multi-Objective Optimization to Workflow Grid Scheduling using Reference Point based Evolutionary Algorithm" in International Journal of Computer Applications (0975 8887) Volume 22 - No.6, May 2011.

[2] Juan J. Durillo, RaduProdan" Multi-objective workflow scheduling in Amazon EC2 " in Springer June 2014, Volume 17, Issue 2, pp 169-189

[3] Sonia Yassa, Rachid Chelouah, Hubert Kadima, and Bertrand Granado" Multi-Objective Approach for Energy-Aware Workflow Scheduling in Cloud Computing Environments "in Scientific World Journal. 2013; 2013: 350934
[4] Topcuoglu, Haluk; Hariri, Salim Wu, M. (2002). "Performance-effective and low-complexity task scheduling for heterogeneous computing". IEEE Transactions on Parallel and Distributed Systems 13 (3): 260-274.

[5] Sanjaya K. Panda1, IEEE Member, Prasanta K. Jana2, IEEE Senior Member " A Multi-Objective Task Scheduling Algorithm for Heterogeneous Multi-Cloud Environment "in Electronic Design, Computer Networks \& Automated Verification (EDCAV), 2015 International Conference on.

[6] J. Li, M. Qiu, Z. Ming, G. Quan, X. Qin and Z. Gu, "Online Optimization for Scheduling Pre-emptable Tasks on IaaS Cloud System",Journal of Parallel Distributed Computing, Elsevier, Vol. 72, pp. 666-677, 2012.

[7] Atul Vikas Lakraa, Dharmendra Kumar Yadav "MultiObjective Tasks Scheduling Algorithm for Cloud Computing Throughput Optimization"

[8] MengXu, Lizhen Cui, Haiyang Wang, Yanbing Bi A Multiple QoS Constrained Scheduling Strategy of Multiple Workflows for Cloud Computing in 2009 IEEE International Symposium on Parallel and Distributed Processing with Applications

[9] Jia Yu, RajkumarBuyya and Chen KhongTham, "CostbasedScheduling of Scientific Workflow Applications on Utility Grids", In 1st IEEE International Conference on e-Science and GridComputing, Melbourne, Australia, Dec. 5-8, 2005.

[10] Ke Liu, Jinjun Chen, Yun Yang and Hai Jin, "sA throughput maximization strategy for scheduling transaction-intensiveworkflows on SwinDeW-G", Concurrency and Computation: Practice and Experience, Wiley, 20(15):1807-1820, Oct. 2008.

[11] Claudia Szabo, Trent Kroeger " Evolving Multi-objective Strategies for Task Allocation of Scientific Workflows on Public Clouds " in WCCI 2012 IEEE World Congress on Computational Intelligence June, 10-15, 2012 Brisbane, Australia

[12] Anil Kumar Gupta, Shashank Shukla, Sandeep Saxena, Sanjay Khakhil "A Journey Towards Workflow Scheduling of Cloud Computing" in International Journal of Computer Applications (0975 - 8887) Volume 123 No.4, August 2015

[13] R.T. Marler and J.S. Arora, "Survey of multi-objective optimization methods in engineering," Structural and Multidisciplinary Optimization, Vol. 26, No. 6, pp. 369395, April 2004.

[14] T. Fahringer, R. Prodan, R. Duan, F. Nerieri, S. Podlipnig, J. Qin, M. Siddiqui, H. L. Truong, A. Villaz'on, and M. Wieczorek, "Askalon: a grid application development and computing environment," in $6^{\text {th }}$ IEEE/ACM International Conference on Grid Computing, pp. 122-131, November 13-14, 2005.

[15] Fan Zhanga,b, JunweiCaob, Keqin Li, Samee U. Khand, Kai Hwange, "Multi-objective scheduling of many tasks in cloud platforms" in Future Generation Computer Systems 37 (2014) 309-320.

[16] Y.C. Ho, R. Sreenivas, P. Vaklili, Ordinal optimization of discrete event dynamic systems, Journal of Discrete Event Dynamic Systems 2 (2) (1992) 61-88. 
[17] Y.C. Ho, Q.C. Zhao, Q.S. Jia, Ordinal Optimization, Soft Optimization for Hard Problems, Springer, 2007.

[18] Juan J. Durillo and VladNae and Radu Prodan "MultiObjective Workflow Scheduling: An Analysis of the Energy Efficiency and Makespan Tradeoff" in 2013 13th IEEE/ACM International Symposium on Cluster, Cloud, and Grid Computing.

[19] H. Topcuoglu, S. Hariri, and M.-Y. Wu, "Performance effective and low-complexity task scheduling for heterogeneous computing," Parallel and Distributed Systems, IEEE Trans. on, vol. 13, no. 3, pp. $260-274$, mar 2002.

[20] M. Mezmaz, N. Melab, Y. Kessaci, Y. Lee, E.-G. Talbi, A.Y.Zomaya, and D. Tuyttens, "A parallel bi-objective hybrid metaheuristic for energy-aware scheduling for cloud computing systems," Journal of Parallel and Distributed Computing, no. 71, pp. 1497-1508, 2011.

[21] J. Kolodziej, S. U. Khan, and F. Xhafa, "Genetic algorithms for energy-aware scheduling in computational grids," in 2011Int. . Conf. on P2P, Parallel, Grid, Cloud and Int. et Computing, 2011

[22] Hamid Mohammadi Fard, RaduProdan, Juan Jose Durillo Barrionuevo and Thomas Fahringer, "A Multi-Objective Approach for Workflow Scheduling in Heterogeneous Environments " in 2012 12th IEEE/ACM International Symposium on Cluster, Cloud and Grid Computing.

[23] Paul Rani, M.Gomathy Nayagam, "Multi-objective Qos Optimization Based on Multiple Workflow Scheduling in Cloud" in International Journal of Innovative Research in Computer and Communication Engineering Vol. 1, Issue 2, April 2013. 